\title{
The Prevalence of Haemoparasitic Infection in Dogs Attending ECWA Vertinary Clinic, Bukuru, Jos South Local Government Area, Plateau State
}

\author{
Nonyelu Maureen Ifeoma \\ Department of Pathology, Delta State University Teaching Hospital, Oghara, Nigeria \\ Email: maureenify@yahoo.com
}

Received January 17, 2013; revised February 17, 2013; accepted March 17, 2013

Copyright (C) 2013 Nonyelu Maureen Ifeoma. This is an open access article distributed under the Creative Commons Attribution License, which permits unrestricted use, distribution, and reproduction in any medium, provided the original work is properly cited.

\begin{abstract}
This study was conducted to investigate the prevalence of haemoparasites of dogs attending ECWA veterinary Clinic, Bukuru, Jos south local government area in Plateau state, Nigeria, and to determine the effective control strategy. From July to September 2011, blood samples of 150 dogs were collected and examined under light microscope. The age, sex and breed of the dogs were also recorded. Wet and unstained blood film shows no motility hence no motile haemoparasite while the Giemsa stained blood smear techniques of both thick and thin film, showed the presence of only Babesia spp in 89 (59.3\%), while 61 (40.7\%) were negative for any haemoparasite.
\end{abstract}

Keywords: Haemoparasite

\section{Introduction}

Parasitology is the area of biology concerned with the phenomenon of dependence of one living organism on another. A parasite is an organism that is entirely dependent on another organism, refer to as its host, for all or part of its life cycle and metabolic requirements [1].

The host is defined as an organism which harbours the parasite and provides the nourishment and shelter to the parasites. Definitive host harbours the adult parasite, the most highly developed form of a parasite or where the parasite replicates sexually. Intermediate host harbours the larval or asexual stages of a parasite. Paratenic host is a host in which larval stage of a parasite survives but does not develop further. Reservoir host is a host that harbours the parasite and serves as an important source to other susceptile hosts. Epidemiologically, reservoir hosts are important in the control of parasitic diseases [1].

In demonstrating this phenomenon of dependence of living organsim on one another, different relationship arises. Some parasites live only on the surface of their hosts and others on the inside. Those on the surface are called external or ectoparasites e.g. lice, fleas, ticks etc. The others are internal parasites or endoparasites e.g. Plasmodium, Babesia etc. Another feature is the distintion between temporary parasites and permanent parasites. Temporary parasites are such specie as the mos- quito and the horsefly, which visit their hosts only when they need the blood of their hosts which is their food. Permanent parasites are parasites throughout or the greater part of their lives. Many temporary parasites are important because in the act of sucking blood, they convey to their hosts the causes of serious diseases. Such causes may be either other parasitic organisms such as the trypanosomes or the malaria parasites or bacteria or viruses. Some parasites, on the other hand, are parasitic only when the opportunity for parasitic life becomes available, this type is called facultative parasites. The contrast is the obligate parasites which must be parasitic always and can live in no other way [2].

A vector is an agent, usually an insect that transmits an infection from one host to another. The term mechanical vector is used to describe a vector which assists in the transfer of parasitic forms between hosts but is not essential in the life cycle of the parasite.

With increased knowledge, it has become known that certain form of parasitic, viral and bacterial infection, although they infect man and often cause serious epidermic, are nevertheless, essentially disease of animals.

\section{Literature Review}

Haemoparasitic infection are parasitic infection, where the parasite itself, or in stage of its development, circu- 
lates in the blood stream. Haemoparasites include the families Haemsporidia (Plasmodium, Haemoproteus, Leucocytozoon), Piroplasms (Babesia), Haemogregarine (Hepatozoon, Haemogregarina), Rickettsias (Aegyptianella, Anaplasma, Ehrlichia, Neorickettsia, Rickettsia, Theileria), Trypanosomes (Trypanosoma, Leishmania), Mycoplasma, Haemobartonella, Dirofilaria and Eperythrozoon.

Some of these haemoparasites are of bacteria origin. Discussion will be based on those that are of protozoa and nematodes origin and common with dogs.

\subsection{Babesia}

The classification of Babesia spp. places them in order Piroplasmida within the phylum Apicomplexa. Two morphologically distinct forms of the erythrocytic stage in the canine host were recognised in early studies that led to the naming of the larger form, measuring approximately $3-5 \mu \mathrm{m}$ as $B$. canis, and the smaller $(1-3 \mu \mathrm{m})$ as $B$. gibsoni [3].

The first suggestion that all B. canis isolates were not identical species came from the German protozoologist Eduard Reichenow who recognised differences in pathogenicity of "B. canis" isolates from France and North Africa (these were most likely parasites that are currently known as B. (canis) canis and B. (canis) vogeli, respecttively) [3].

"Babesia canis" was reclassified into three sub-species (B. canis canis, B. canis rossi and B. canis vogeli) on the basis of cross-immunity, serological testing, vector specificity and molecular phylogeny [3].

Canine babesiosis is a common and clinically signifycant tick-borne haemoprotozoan disease with a worldwide distribution. Babesia species are often referred to as piroplasms, a collective term for phenotypically similar protozoan parasites that utilise mammalian erythrocytes in their life cycle. Piroplasms of domestic animals encompass two main genera, Babesia and Theileria, and have been subject of intense research interest and molecular-based re-classification during the last 10 years. In dogs, infection by these haemoparasites results in a wide range of clinical presentations; from subclinical disease to serious illness characterised by fever, pallor, jaundice, splenomegaly, weakness and collapse associated with intra- and extravascular haemolysis, hypoxic injury, systemic inflammation, thrombocytopenia and pigmenturia. Although canine babesiosis is recognised as a tick-borne disease, transmitted by a variety of well-described Ixodid vectors around the world, Babesia gibsoni is an emerging disease with molecular evidence of clonal expansion due to non-vectored transmission by blood exchange during fighting and biting. Recent research into canine babesiosis has focussed on determining the taxonomic status of well recognised and newly discovered canine piroplasms, developing improved diagnostic methods, investigating aspects of pathophysiology and searching for improved chemotherapeutic and immunoprophylactic protocols [3].

\subsection{Trypanosomes}

Trypanosomiasis or trypanosomosis is the name of several diseases in vertebrates caused by parasitic protozoan trypanosomes of the genus Trypanosoma. Approximately 500,000 men, women, and children in 36 countries of sub-Saharan Africa suffer from human African trypanosomiasis which is caused by either Trypanosoma brucei gambiense or Trypanosoma brucei rhodesiense. The other human form of trypanosomiasis, called Chagas disease, causes 21,000 deaths per year (mainly in Latin America) [4].

Trypanosome, a member of a group of protozoa (singlecelled animals) that are blood parasites. They are the cause of diseases, such as African sleeping sickness and Chagas' disease in human beings. In cattle and other animals, serve as the reservoir for the protozoa, the disease is called nagana. Two variations of the disease occur in central and western Africa, both of them transmitted in the salivary glands of infected tsetse flies. The most common is caused by T. brucei gambiense, whereas a more local version is caused by T. brucei rhodesiense. In South America, another version of the protozoan, T. cruzi, is transmitted by the triatoma bug and it causes Chagas' disease [5].

Trypanosome is transmitted from one human being to another via the tsetse fly, a blood-sucking fly found only in Africa. The trypanosome starts as a parasite in the blood, but in the later stages of the infection can invade the central nervous system, causing an inflammation of the brain and spinal cord that is responsible for the characteristic neurological symptom of sleeping [5]. The trypanosome is never transmitted directly from one person to another. Because of this, the spread of the disease is prevented by destroying the tsetse fly.

\subsection{Leishmania}

Leishmania is a genus of Trypanosomatid protozoa, and is the parasite responsible for the disease leishmaniasis [6]. It is spread through sandflies of the genus Phlebotomus in the Old World, and of the genus Lutzomyia in the New World. Their primary hosts are vertebrates; Leishmania commonly infects hyraxes, canids, rodents, and humans. Leishmania currently affects 12 million people in 98 countries.

The biochemistry and cell biology of Leishmania is similar to that of other kinetoplastids. They share the same main morphological features; a single flagellum which has an invagination, the flagellar pocket, at its base, a 
kinetoplast which is found in the single mitochondrion and a sub-pelicular array of microtubles which make up the main part of the cytoskeleton.

Leishmania possess a lipophosphoglycan coat over the outside of the Leishmania cell. Lipophosphoglycan is a trigger for Toll-like receptor 2, a signalling receptor involved in triggering an innate immune response in mammals.

The precise structure of lipophosphoglycan varies depending on the species and life cyclestage of the parasite. The glycan component is particularly variable and different lipophosphoglycan variants can be used as a molecular marker for different life cycle stages. Lectins, a group of plant proteins which bind different glycans, are often used to detect these lipophosphoglycan variants. For example peanut agglutinin binds a particular lipophosphoglycan found on the surface of the infective form of Leishmania major.

Leishmania cells have two morphological forms: promastigote (with an anteriorflagellum in the insect host, and amastigote (without flagella) in the vertebrate host. Infections are regarded as cutaneous, mucocutaneous, or visceral.

Leishmaniasis is still the cause of a huge burden on public health worldwide. Sand flies (Phlebotominae) are the only accepted biological vectors of Leishmania species. These small flies ingest amastigote forms of the parasite while feeding on a reservoir host and, they provide a suitable environment for the development of promastigote infective forms in their gut. Yet, this history is not so simple; not all sandfly species are able to transmit all Leishmania species. Indeed, there is a close relation between particular sandflies and certain Leishmania species, which seems to be a result of a long evolutionary history [7].

Recently, a typical case of canine visceral leishmaniasis by Leishmania amazonensis has been diagnosed in Brazil, in an area where the specific vector (i.e. Lutzomyia flaviscutellata) is absent. Nonetheless, Lutzomyia longipalpis is present and has been regarded as a supputative vector because this sandfly supports the development of L. amazonensis in the laboratory. Additionally, Lutzomyia migonei has been regarded as a supputative vector of $L$. infantum in certain areas of northern Argentina and north eastern Brazil, where the specific vector, $L$. longipalpis, is apparently absent. Indeed, this concept is of practical interest, because a permissive vector could play a role in the epidemiology of leishmaniasis in areas where the specific vector is absent [7].

Lipophosphoglycan is used by the parasite to promote its survival in the host and the mechanisms by which the parasite does this center around modulating the immune response of the host. This is vital as the Leishmania parasites live within macrophages and need to prevent the macrophage from killing them. Lipophosphoglycan has a role in resisting the complement system, inhibiting the oxidative burst response, inducing an inflammation response and preventing natural killer $\mathrm{T}$ cells recognising that the macrophage is infected with the Leishmania parasite [8].

It is acknowledged that in "specific vectors" the protozoa attach themselves to midgut lipophosphoglycan (LPG) receptors, a fundamental step to avoid expulsion from the midgut when the sandfly defecates. Most recently, it was demonstrated that some sandflies "permissive vectors" support development of multiple Leishmania species, which might adopt alternative pathways (LPG-independent) to survive in the sandfly's midgut [9].

\subsection{Dirofilaria}

Two subgenera, Dirofilaria (Dirofilaria) species and Dirofilaria (Nochtiella) species are recognized. Dirofilaria (Dirofilaria) immitis is the most frequent agent of pulmonary dirofilariasis. Although D. (Dirofilaria) immitis primarily causes lung lesions, it has, on rare occasion caused intra-abdominal infection and even subcutaneous nodules.

These worms are natural parasites of other, non-human, mammals. In humans, there are two forms of the disease:

1) pulmonary dirofilariasis caused primarily by Dirofilaria immitis (the dog heartworm), and

2) subcutaneous dirofilariasis caused primarily by $D$. tenuis and $D$. repens, parasites of the raccoon and of dogs and cats respectively; and by D. ursi from the bear [10].

Humans are poor hosts for all Dirofilaria species such that in humans, the worm usually dies before reaching sexual maturity and does not release viable microfilariae.

Dirofilaria immitis is a common parasite of dogs (and some other carnivores) in many parts of the world. It is a considerable veterinary problem, particularly in the USA and Japan. In the dog, adult worms lie coiled in tangled masses in the right ventricle of the heart. The microfilariae circulate in the blood and are transmitted by mosquitoes.

The parasite is commonly called "heartworm"; however, that is a misnomer because the adults actually reside in the pulmonary arterial system (lung arteries) for the most part, and the primary effect on the health of the animal is a manifestation of damage to the lung vessels and tissues. Occasionally, adult heartworms migrate to the right heart and even the great veins in heavy infections. Heartworm infection may result in serious disease for the host [11].

The female measures $25-30 \mathrm{~cm}$ in length and about 1 $\mathrm{mm}$ in width. The male is smaller and measures $12-18$ $\mathrm{cm}$ in length. Microfilariae are unsheated, $300-325 \mu \mathrm{m}$ in length and about $7 \mu \mathrm{m}$ in width. They are found in the 
blood, where they are ingested by mosquitoes [1].

Dog is the definitive host and the mosquitoes are intermediate hosts. The infective larval forms, from the proboscis of the mosquito, are injected into the subcutaneous tissue of the dog by the bite of mosquito. The larvae enter into the ciculation and ultimately find their way to the heart, where they develop into adults. The diagnosis in dogs may be made by observing circulating microfilariae in the peripheral bloood [1].

\section{Materials and Method}

\subsection{Materials}

All materials used were commercially acquired except staining rack which are available in the laboratory.

\subsection{Samples}

Blood samples were aseptically collected from 150 dogs (different breeds) attending ECWA Veterinary Clinic, Bukuru.

\subsection{Method of Collection of Samples}

A swab was used to sterilize a site of cephalic vein on the fore limb of the dogs while the mouth had been tied with mouth gag (Figure 1). A sterile $2 \mathrm{ml}$ syringe and hypodermic needle was used and a small quantity of blood was drawn out from the vein of each dog and dispensed into an EDTA (Ethylene diamine tetraacetic acid) container, mixed and labelled accordingly. The age, sex and breed of the dogs were also recorded.

\subsection{Method of Processing Samples}

\subsubsection{Wet Preparation}

Wet preparation of samples were done by placing a drop of well mixed blood on a clean grease free slide and cov-

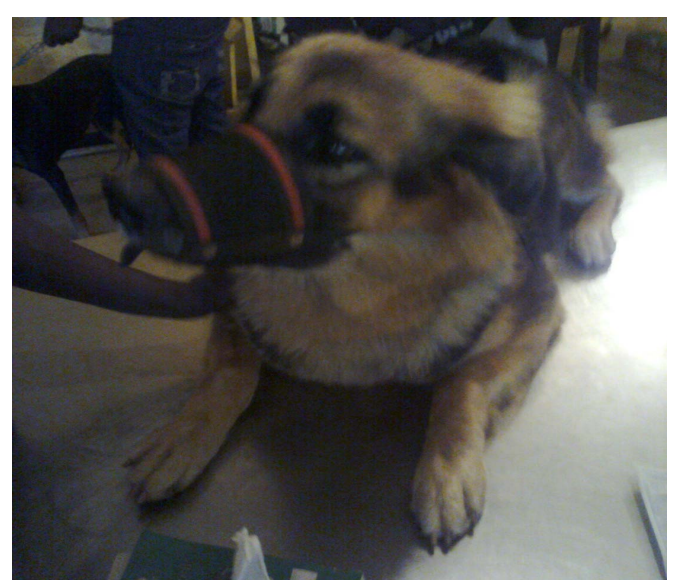

Figure 1. Mouth of a German Shephered Dog tied with a gag and about to collect blood sample from the cephalic vein [12]. ered with a coverslip, avoid air bubble. This was done to screen for the presence of motile haemoflagellates.

\subsubsection{Thick Film}

Thick film was made by placing a large drop of well mixed blood onto a clean grease free slide, and spread with the edge of another slide in a circular motion. The blood was continously stirred for about 30 seconds to prevent formation of fibrin clots. It was then air-dried.

\subsubsection{Thin Film}

Thin film of the blood sample was made by placing a drop of well mixed blood onto a clean grease free slide at about $2 \mathrm{~cm}$ from the right end. The drop of blood was touched with the edge of another slide, and held at an angle of 30 degrees and pushed in a quick but gently move such that a head, body and tail were made.

\subsection{Staining Technique}

The dried thin blood film was fixed with pure methanol or ethanol for 1 minute and allowed to dry. The Giemsa stain used was diluted 1:10 in buffered distilled water to a $\mathrm{pH}$ of 7.2. The diluted stain was poured over the film (thick and thin) on the slide and left to stand for 30 minutes. The stained slide was then flushed in a gentle flow of buffered distilled water with $\mathrm{pH}$ 7.2, after which it was placed in an upright position to drain and dry. The stained film was examined under oil-immmersion lens.

\section{Result}

One hundred and fifty blood samples were collected from seven breeds of dogs which are Alsatian, Boar Bull, Bull Mastiff, Caucasian, Mongreal (Local), Poodle, and Rottweiler, both male and female from different age range, processed and examined under light microscope. Wet blood films show no motile haemoparasite while thin and thick blood smear techniques showed the presence of only Babesia spp in 89 dogs while 61 dogs had a normal uninfected red blood cells (Plates 1 and 2).

Table 1, shows that Babesia sp. appear to be more prevalent than other haemoparasite. The dogs negative for haemoparasite are $(61 \%)$ [3].

Table 2 shows the age groups of the different breeds of dogs sampled [3].

In Table 3, the distribution of those infected breeds of dogs with Babesia sp. shows that it is not prevalent within a particular age and breeds. The prevalence was higher in local breed "Mongreal" than in other exotic breeds [3].

It affects all sexs and in respective of the breed [3].

\section{Discussion}

Of the one hundred and fifty dogs sampled, 89 dogs were 
Table 1. Prevalence of the haemoparasites studied.

\begin{tabular}{|c|c|c|c|c|c|c|c|c|c|c|c|c|c|c|c|c|}
\hline \multirow{2}{*}{ Breed } & \multicolumn{2}{|c|}{ SEX } & \multicolumn{8}{|c|}{ AGE } & \multirow{2}{*}{$\begin{array}{l}\text { Wet } \\
\text { Film }\end{array}$} & \multicolumn{5}{|c|}{ Thick And Thin Smear } \\
\hline & Male & Female & $\begin{array}{c}0-3 \\
M\end{array}$ & $\begin{array}{c}\text { Mths } \\
\text { F }\end{array}$ & $\begin{array}{c}4-6 \\
M\end{array}$ & $\begin{array}{c}\text { Mths } \\
\text { F }\end{array}$ & $\begin{array}{c}7 \text { - } 9 \\
M\end{array}$ & $\begin{array}{c}\text { Mths } \\
\text { F }\end{array}$ & $\begin{array}{l}\geq 1 Y \\
M\end{array}$ & $\begin{array}{r}\text { Year } \\
\mathbf{F}\end{array}$ & & Babesia. spp & Trypa. spp & Leishm. spp & Dirofil. spp & Nil \\
\hline Alsatian & 8 & 17 & 2 & 1 & 2 & 2 & 2 & 6 & 2 & 8 & NIL & 17 & NIL & NIL & NIL & 8 \\
\hline Boar Bull & 3 & 8 & 0 & 1 & 1 & 1 & 1 & 2 & 1 & 4 & NIL & 5 & NIL & NIL & NIL & 6 \\
\hline Bull Mastiff & 9 & 5 & 1 & 1 & 3 & 0 & 3 & 2 & 2 & 2 & NIL & 7 & NIL & NIL & NIL & 7 \\
\hline Caucasian & 12 & 19 & 3 & 1 & 1 & 1 & 4 & 3 & 4 & 14 & NIL & 13 & NIL & NIL & NIL & 18 \\
\hline Mongreal & 21 & 17 & 4 & 2 & 7 & 5 & 2 & 4 & 8 & 6 & NIL & 29 & NIL & NIL & NIL & 9 \\
\hline Poodle & 2 & 5 & 0 & 0 & 0 & 2 & 1 & 2 & 1 & 1 & NIL & 3 & NIL & NIL & NIL & 4 \\
\hline Rottweiler & 9 & 15 & 1 & 2 & 1 & 1 & 3 & 6 & 4 & 6 & NIL & 15 & NIL & NIL & NIL & 9 \\
\hline Total & 64 & 86 & & 9 & 2 & 7 & & 41 & 63 & & & 89 & & & & 61 \\
\hline
\end{tabular}

Key: Nil-no heamoparasite was seen; $\geq 1$-1 year and above; Trypa. spp-Typanosomes specie; Leishm. spp-Leishmania specie; Dirofil. spp-Dirofilaria specie.

Table 2. Age distribution amongs the different breeds of dogs.

\begin{tabular}{|c|c|c|c|c|}
\hline \multirow{2}{*}{ Breeds } & \multicolumn{4}{|c|}{ Ages Sampled } \\
\hline & $0-3$ Months & 4- 6 Months & 7 - 9 Months & $\leq 1$ Months \\
\hline Alsatian & $3(2 \%)$ & $4(2.6 \%)$ & $8(5.3 \%)$ & $10(6.6 \%)$ \\
\hline Boar Bull & $1(0.6 \%)$ & $2(1.3 \%)$ & $3(2 \%)$ & $5(3.3 \%)$ \\
\hline Bull Mastiff & $2(1.3 \%)$ & $3(2 \%)$ & $5(3.3 \%)$ & $4(2.6 \%)$ \\
\hline Caucasian & $4(2.6 \%)$ & $2(1.3 \%)$ & $7(4.6 \%)$ & $18(12 \%)$ \\
\hline Mongreal & $6(4 \%)$ & $12(8 \%)$ & $6(4 \%)$ & $14(9.3 \%)$ \\
\hline Poodle & $0(0 \%)$ & $2(1.3 \%)$ & $3(2 \%)$ & $2(1.3 \%)$ \\
\hline Rottweiler & $3(2 \%)$ & $2(1.3 \%)$ & $9(6 \%)$ & $10(6.6 \%)$ \\
\hline Total & $19(12.7 \%)$ & $27(18 \%)$ & $41(27.3 \%)$ & $63(42 \%)$ \\
\hline
\end{tabular}

Table 3. Distribution of infected dogs in relation to their ages.

\begin{tabular}{|c|c|c|c|c|}
\hline \multirow{2}{*}{ Breeds } & \multicolumn{4}{|c|}{ Ages Sampled } \\
\hline & 0 - 3 Months & 4- 6 Months & 7 - 9 Months & $\leq 1$ Months \\
\hline Alsatian & $1(0.6 \%)$ & $2(1.3 \%)$ & $6(4 \%)$ & $8(5.3 \%)$ \\
\hline Boar Bull & $0(0 \%)$ & $1(0.6 \%)$ & $1(0.6 \%)$ & $3(2 \%)$ \\
\hline Bull Mastiff & $1(0.6 \%)$ & $1(0.6 \%)$ & $3(2 \%)$ & $2(1.3 \%)$ \\
\hline Caucasian & $2(1.3 \%)$ & $0(0 \%)$ & $3(2 \%)$ & $8(5.3 \%)$ \\
\hline Mongreal & $3(2 \%)$ & $9(6 \%)$ & $6(4 \%)$ & $11(7.3 \%)$ \\
\hline Poodle & $0(0 \%)$ & $0(0 \%)$ & $2(1.3 \%)$ & $1(0.6 \%)$ \\
\hline Rottweiler & $1(0.6 \%)$ & $1(0.6 \%)$ & $5(3.3 \%)$ & $8(5.3 \%)$ \\
\hline Total & $8(5.3 \%)$ & $14(9.3 \%)$ & $26(17.3 \%)$ & $41(27.3 \%)$ \\
\hline
\end{tabular}

positive for Babesia specie of parasite with the thick and thin film staining technique while 61 dogs were without any form of haemoparasites in their blood sample. All the age groups of these dogs were infected with these protozoan parasites (Tables 1-4 and Figure 2).

In agreement with previous studies [3], age, sex and 
Table 4. Comparism of infection rate between males and females with babesia specie with those not infected with any haemoparasites.

\begin{tabular}{|c|c|c|c|c|}
\hline \multirow{2}{*}{ Breeds } & \multicolumn{2}{|c|}{ Total of both sexs } & \multirow{2}{*}{$\begin{array}{c}\text { Babesia } \\
\text { spp }\end{array}$} & \multirow{2}{*}{ No haemoparasite } \\
\hline & M & $\mathrm{F}$ & & \\
\hline Alsatian & 8 & 17 & $17(11.3 \%)$ & $8(5.3 \%)$ \\
\hline Boar Bull & 3 & 8 & $5(3.3 \%)$ & $6(4 \%)$ \\
\hline Bull Mastiff & 9 & 5 & $7(4.7 \%)$ & $7(4.7 \%)$ \\
\hline Caucasian & 12 & 19 & $13(8.7 \%)$ & $18(12 \%)$ \\
\hline Mongreal & 21 & 17 & $29(19.3 \%)$ & $9(6 \%)$ \\
\hline Poodle & 2 & 5 & $3(2 \%)$ & $4(2.7 \%)$ \\
\hline Rottweiler & 9 & 15 & $15(10 \%)$ & $9(6 \%)$ \\
\hline Total & \multicolumn{2}{|c|}{150} & $89(59.3 \%)$ & $61(40.7 \%)$ \\
\hline
\end{tabular}

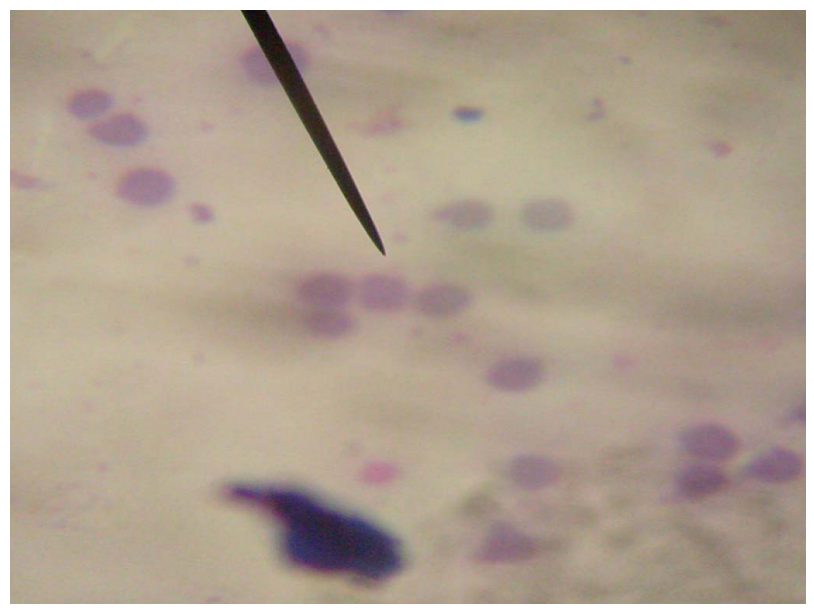

Plate 1. The normal red blood cell in dogs shows a central palor [1].

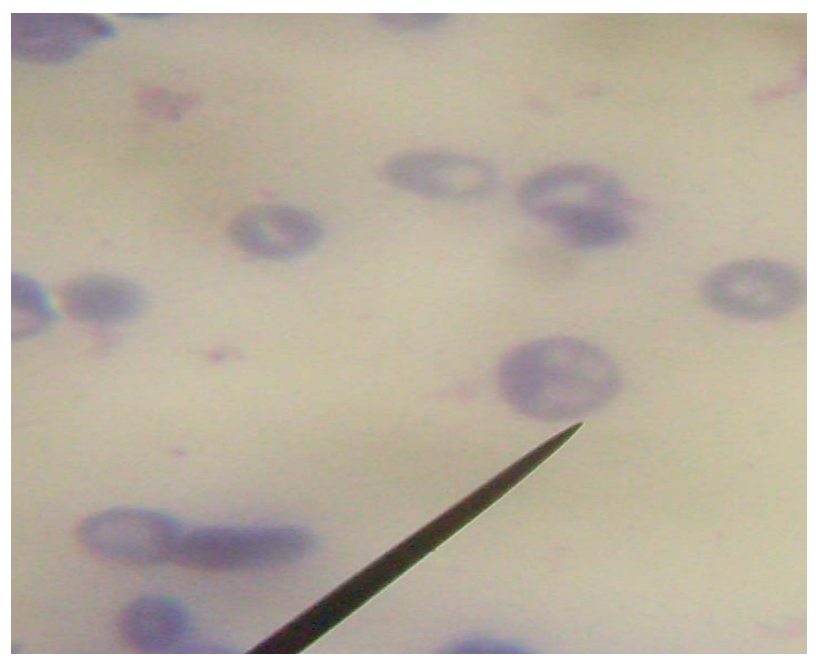

Plate 2. Babesia spp. present in the red blood cell of infected dog showing the presence of a tetrad forms "Maltese cross" [1].

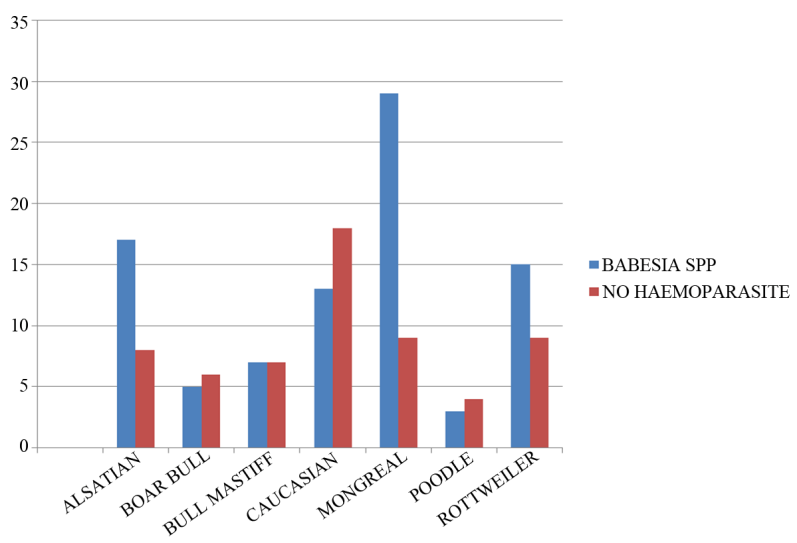

Figure 2. Comparism of the total number infected with Babesia specie with those not infected with any haemoparasites [3].

breeds of the animals did not have significant influence on the prevalence of haemoparasites. Also observed, all the spp of these dogs were not spared of the infections even though the parasitic density were low.

Ixodid ticks of the genera Hyalomma, Boophilus, Dermacentor and Rhipicephalus have been identified as vectors for the transmission of Babesia protozoa to natural host [13]. All stages (that is the larva, nymph and adult) of the Ixodid ticks were seen on some of these dogs while some didn't have any on them.

Dogs are household pets, which have now become part of the family structure, increasing the risk of transmissible diseases between pets and family members. Man can acquire infection when the salivary babesial sporozoites are inoculated by the feeding vector tick, which leads to a zoonotic infection.

\section{Conclusion}

Since the appearance of Babesia spp in Giemsa stained 
film can not be distinguish from Plasmodium falciparum or is mistaken for Plasmodium falciparum rings, (Plate II ) "although the presence of tetrad form in the red blood cells and the absence of parasitic haemazoin ( malaria pigment ) are diagnostic for Babesia spp.”, dogs infested with ticks should properly be treated to prevent these zoonotic infection which destroys the red blood cells.

\section{Recommendation}

Dogs owners should take good care of them by regularly dipping or spraying them with insecticide which is either in powder or liquid form.

Those incriminated with Babesiosis must be completely treated.

In as much as green grassy areas serve as momentary habitats for ticks in their life-cycle development, absolute care should be enforced to prevent and control overgrowth, hence regular use of pesticides/insecticides.

\section{Acknowledgements}

This project report is dedicated to GOD ALMIGHTY through JESUS CHRIST my LORD for HIS guidance, provision, protection and wisdom through out this project.

\section{REFERENCES}

[1] D. R. Arora and B. B. Arora, "Medical Parasitology,"3rd Edition, 2010.

[2] G. O. Ariyibi, "A Textbook of Human Parasitology," 2001.
[3] P. J. Irwin, "Canine Babesiosis: From Molecular Taxonomy to Control," Murdoch University, Murdoch, Parasites \& Vectors, Vol. 2, No. 1, 2009, p. S4. doi:10.1186/1756-3305-2-S1-S4

[4] J. D. Maya, B. K. Cassels and P. Iturriaga-Vásquez, "Mode of Action of Natural and Synthetic Drugs against Trypanosoma cruzi and Their Interaction with the Mammalian Host," Comparative Biochemistry and Physiology Part A: Molecular \& Integrative Physiology, Vol. 146, No. 4, 2007, pp. 601-620. doi:10.1016/i.cbpa.2006.03.004

[5] Microsoft ${ }^{\circledR E n c a r t a} \AA 2009$. C 1993-2008 Microsoft Corporation.

[6] P. Myle and N. Fasel, "Leishmania: After the Genome," Caister Academic Press, Norfolk, 2008.

[7] F. Dantas-Toress, "Ticks as Vectors of Leishmania Parasites," 2011.

[8] S. Kamhawi, "Phlebotomine Sandflies and Leishmania Parasites: Friends or Foes?" Trends in Parasitology, Vol. 22, 2006, pp. 439-445. doi:10.1016/j.pt.2006.06.012

[9] P. Volf and J. Myskova, "Sandflies and Leishmania: Specific Versus Permissive Vectors," Trends in Parasitology, Vol. 23, 2007. pp. 91-92. doi:10.1016/j.pt.2006.12.010

[10] A. M. Marty and R. C. Neafie, "Dirofilariasis: Pathology of Infectious Diseases Volume 1 Helminthiases," 2000.

[11] S. J. Ettinger and E. C. Feldman, "Textbook of Veterinary Internal Medicine," 7th Edition, Saunders Company, Philadelphia, 2010.

[12] K. M. Dyce, “Textbook of Veterinary Anatomy,” 2002.

[13] E. J. L. Soulsby, "Helminthes, Arthropods and Protozoa of Domestic Animals," 7th Edition, Bailliere Tindall, London, 1982. 